\title{
The significance of the concordance rate for Type 1 (insulin-dependent) diabetes in identical twins
}

\author{
P.Olmos, R. A'Hern, D.A. Heaton, B. A. Millward, D. Risley, D. A. Pyke and R. D.G. Leslie \\ Departments of Medicine and Diabetes, Kings College Hospital, London, UK
}

\begin{abstract}
Summary. We studied prospectively 49 non-diabetic identical twins of recently-diagnosed Type 1 (insulin-dependent) diabetic patients for up to 24 years (median 9 years). During this time 15 developed Type 1 diabetes. Actuarial analysis indicates that by 12 years $34 \%$ of the twins will have developed Type 1 diabetes and that thereafter only another $2 \%$ will do so. Inevitable bias in ascertainment of the twins makes it likely that the true figure is less. We conclude that factors which are not genetically determined must be important in
\end{abstract}

the pathogenesis of the disease. The rates of developing Type 1 diabetes in the co-twins declines sharply in the years after diagnosis of the index twin, which suggests that the initiation of the process leading to Type 1 diabetes occurs within a finite, and not a prolonged, period.

Key words: Concordance, identical twins, Type 1 (insulin-dependent) diabetes.
We have attempted to determine the true concordance rate for Type 1 (insulin-dependent) diabetes in identical twins. Previously, our own and other studies have suggested a maximum figure of about $50 \%$, though an actuarial analysis has never been performed on the data [1-4]. In view of the major ascertainment biases in all previous diabetic twin studies including our own it has not been possible to reach a firm conclusion that twin pairs can remain discordant for diabetes irrespective of the period of follow-up. Concordance for Type 1 diabetes in identical twins could be due to shared genetic and/or environmental factors but discordance must be due to non-genetically determined factors. We know that Type 1 diabetes is partly determined by genetic factors because of its association with T-cell receptor genes and genes in the HLA region $[5,6]$, but non-genetic factors such as viruses have also been implicated [7]. We now report a twin study of sufficient size and duration to enable us to perform an actuarial analysis and thereby assess the importance of non-genetically determined factors in Type 1 diabetes.

If Type 1 diabetes is caused by an environmental factor, it might be caused by either prolonged or brief exposure to it. Prolonged exposure or susceptibility to such a factor would mean that the disease rate should rise with time of exposure, as the incidence of lung cancer rises with increasing duration of smoking [8]. On the other hand, if exposure or susceptibility was brief, disease incidence would not rise progressively, but remain constant or fall, as in infectious diseases.

\section{Subjects and methods}

As there is no twin register in Britain a systematic survey of all twins is not possible. We have been collecting data on identical twins, one or both of whom has Type 1 diabetes, for over 20 years. They were ascertained because they were Type 1 diabetic patients, not because they were twins [4]. We do not believe we have ascertained all the identical twins in Britain (one or both of whom has Type 1 diabetes) because, firstly, we estimate that there should be at least 600 twin pairs with Type 1 diabetes in the country, assuming a frequency of one pair of identical twins in every 250 births and a prevalence of Type 1 diabetes of 1:400, yet we have only 176 twin pairs with the disease in our whole series; and secondly, geographical distribution of the twins is uneven. If ascertainment of Type 1 diabetic twins is incomplete, the proportion of concordant and discordant twin pairs is likely to be biased, presumably towards the ascertainment of concordant pairs, as they have a double chance of recognition. In an attempt to discover the true concordance rate for Type 1 diabetes in identical twins, we have confined the present study to pairs in which the twins fulfilled the following criteria: (1) they were ascertained within one year of diagnosis of diabetes in the index twin (the first diabetic twin to be ascertained) irrespective of whether they were then concordant or discordant and (2) the index twin had Type 1 diabetes [9]. Ideally, the study should have begun as soon as one twin of a pair developed Type 1 diabetes; but this has not always been possible.

Of our whole series of identical twins one or both of whom has Type 1 diabetes, 49 pairs fulfilled the criteria for selection. All the index twins were also the first twins of each pair to be diagnosed diabetic. The twins were followed until 1 January 1987, when they 
had been observed for 2 to 24 years (median 9 years). None of the twins were lost to follow-up because they died. The median age of the index twin at the time of diagnosis was 18 years (range 5 to 44 years). Type 1 diabetes was diagnosed by a history of diabetic symptoms, elevated blood glucose, ketonuria and dependence on insulin [9]. Monozygosity was established by typing for blood groups $\mathrm{ABO}, \mathrm{CDE}, \mathrm{MN}, \mathrm{S}, \mathrm{P}, \mathrm{Lu}^{\mathrm{a}}, \mathbf{K}, \mathrm{Le}^{\mathrm{a}}$ and $\mathrm{Fy}^{\mathrm{a}}$ which establishes identity with not more than a $3 \%$ chance of error [4].

\section{Blood glucose}

Whole blood glucose was measured (potassium ferricyanide or glucose oxidase method) either random or fasting and following an oral glucose load in the non-diabetic twins in order to exclude diabetes according to standard criteria [9].

\section{HLA-DR typing}

Whole blood $(10 \mathrm{ml})$ was collected in $8 \mathrm{ml}$ tissue culture medium 199 (Wellcome Beckenham, UK) with the addition of $2 \mathrm{ml}$ sodium citrate $3.8 \%$. After collection the samples were analysed within $24 \mathrm{~h}$ using a two-colour fluorescence technique. All 10 officially recognised DR specificities were defined using a minimum of 60 antisera [6]; HLA genotyping was performed using HLA DR Beta probes [11].

\section{Statistical analysis}

The twins were followed until the closing date or until they developed Type 1 diabetes. Curves showing the percentage of twins who did not develop Type 1 diabetes were calculated by standard actuarial life-table methods [10]. The standard error of a point estimate was computed by use of the Greenwood formula. The risk of developing Type 1 diabetes decreases with time so a Weibull model was considered most appropriate in order to fit a curve to the set of proportions of twins surviving without having developed the disease [10]. This model was then used to determine the proportion of twins remaining non-diabetic when: (1) the median period of follow-up was 12 years and (2) the median age at follow-up was 30 years. The period of follow-up was selected because of those 78 twin pairs who became concordant for Type 1 diabetes in our series of twins; $94 \%$ had done so within 12 years of the diagnosis of their index twin [4]. The age of 30 at follow-up was selected because the onset of Type 1 diabetes is usually before this age.

\section{Results}

Of the 49 twin pairs, 4 were already concordant when they were referred; in a further 11 pairs the second twin developed Type 1 diabetes during the study. The median period between diagnosis of Type 1 diabetes in the first and second twin was 1 year (range 0.1 to 6.0 years). All the twins who became diabetic developed Type 1 diabetes.

A curve was fitted to the proportion of twins whe did not develop Type 1 diabetes, which predicted that at 1,5 , and 10 years follow-up 14,25 , and $32 \%$ of the twins, respectively, would develop the disease. The observed rates of concordance at 1,5 , and 10 years follow-up were 14, 25, and 34\% respectively (see Figure and Table). The predicted proportion of twins developing Type 1 diabetes after 12 years follow-up

NUMBER AT RISK

$\begin{array}{lllllllllllllllll}49 & 42 & 38 & 35 & 31 & 28 & 24 & 19 & 18 & 16 & 16 & 14 & 10 & 10 & 9 & 8 & 7\end{array}$

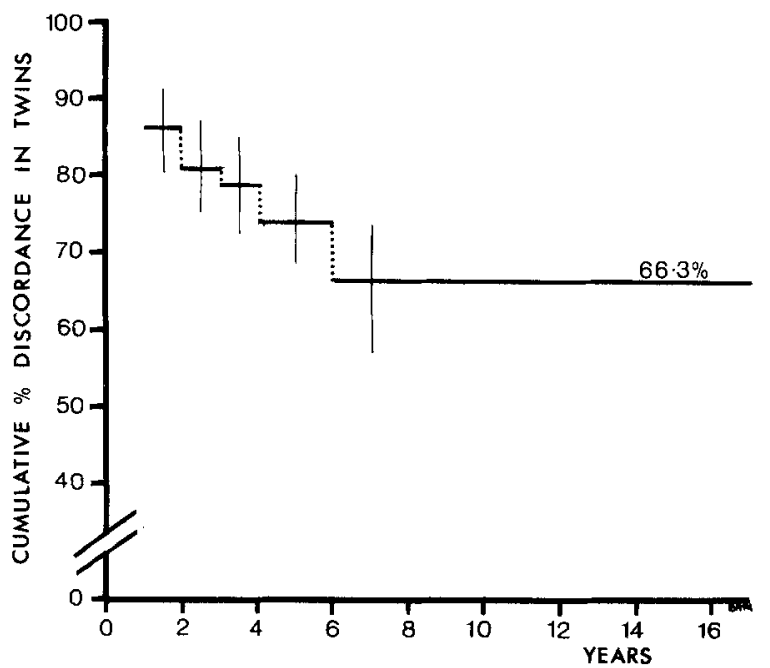

Fig. 1. Life Table showing percentage (with standard error) of identical twins of Type 1 (insulin-dependent) diabetic patients who remain non-diabetic

Table 1. Details of actuarial analysis of a cohort of 49 non-diabetic twins up to 12 years of follow-up

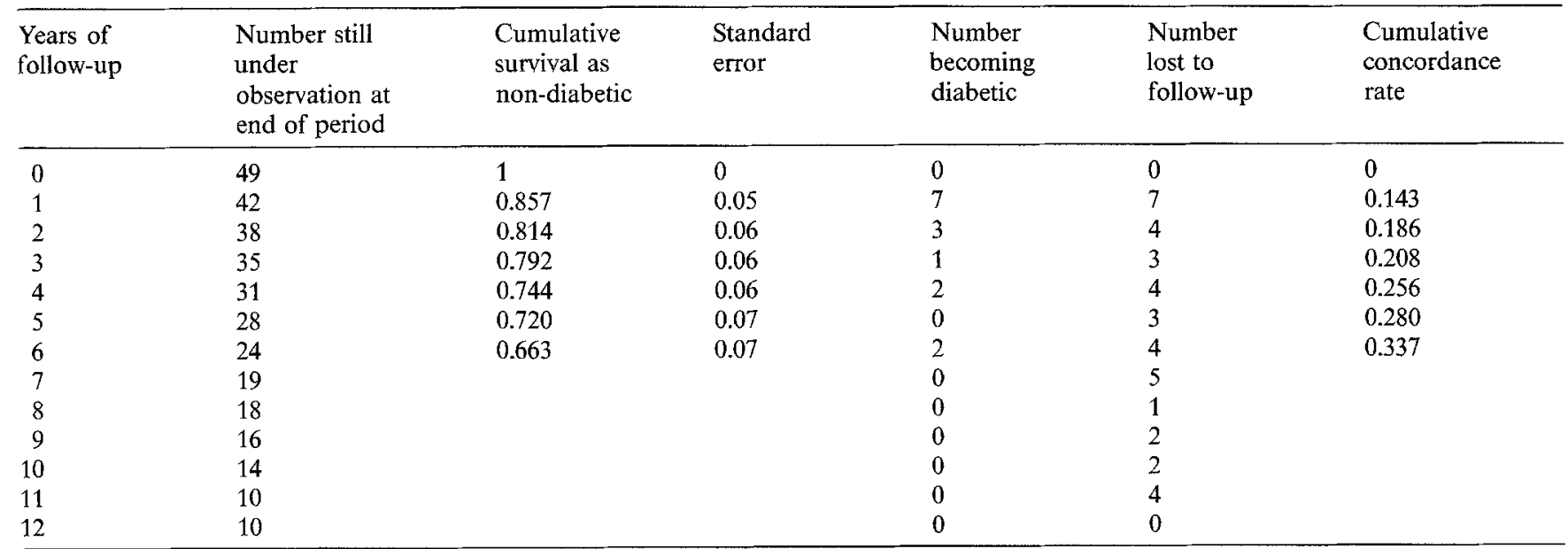


and at a median age of 30 is $34 \%$. The numbers of twins are too small to give confidence intervals for these results.

The rate of developing Type 1 diabetes fell with time from the diagnosis of the index twin (Table 1); by four years $26 \%$ had developed the disease as compared with a further $8 \%$ in the next four years (using Chi-square; $p<0.01$ ).

If we divide the series by age at diagnosis of Type 1 diabetes in the index twin we find that of those 21 twins diagnosed under the age of 15 years, 10 of the co-twins developed Type 1 diabetes; while of the 28 index twins diagnosed over the age of 15 years only 5 of the co-twins developed the disease. Log-rank-analysis was used to analyse the time to onset of diabetes in the second twin of these two groups; the difference was statistically significant (using Chi-square; $p<0.05$ ).

In two of the 15 twin pairs who became concordant the twins were living apart at the time of diagnosis of the index twin as compared with 11 of the 34 pairs who remain discordant (not significant).

Of the 34 twins who remain non-diabetic 26 were typed for HLA-DR; of these 26 twins, $4(15 \%)$ were HLA-DR 3 and 4, $24(92 \%)$ were HLA-DR 3 or 4 . Of the 15 twins who became diabetic 13 were typed for HLA-DR, of whom $6(46 \%)$ were HLA-DR 3 and 4 and all $13(100 \%)$ were HLA-DR 3 or 4 . The numbers of twins are too small to make an actuarial analysis according to HLA type. The distribution of HLA-DR types in these twins is similar to that in Type 1 diabetic patients in general [6].

\section{Discussion}

In this cohort of 49 identical twins of recently-diagnosed Type 1 diabetic patients followed for up to 24 years, 15 developed Type 1 diabetes, in all cases within six years of the diagnosis of their index twin. Actuarial analysis suggests that no more than $36 \%$ of the twins will ever become diabetic. Discordance for Type 1 diabetes between identical twins indicates that the disease must be due, at least in part, to non-genetically determined factors.

The model we have derived from actuarial analysis of the cohort of twins predicts that $34 \%$ of twins will develop Type 1 diabetes when the median age at follow-up is 30 years and the median follow-up is 12 years. Whilst some twins may subsequently develop Type 1 diabetes our observations on those 78 pairs from the whole study who became concordant for Type 1 diabetes shows that $94 \%$ had done so by this time [4]. Thus, calculating from the $34 \%$ of twins who developed Type 1 diabetes by 12 years it might be expected that another $2 \%$ would develop the disease with further follow-up. However, some allowance should be made for the theoretical possibility that other pairs, not under observation, became concordant after 12 years which might slightly alter the true concordance rate. We estimate that the final concordance rate in our series will be not more than $36 \%$. This contrasts with a figure of about $10 \%$ for non-identical twin pairs and for siblings, though using a different method of estimation [3, 12].

We have tried to limit the bias towards ascertaining concordant pairs by considering only those pairs referred within one year of the diagnosis of Type 1 diabetes in the index twin. Nevertheless, 4 twin pairs were already concordant when they were ascertained; these twins might represent a bias towards ascertainment of concordant pairs which could falsely increase the concordance rate. In addition, there must be many twin pairs who were not ascertained by us; and since these pairs are more likely to be discordant than concordant this bias would also increase the concordance rate in our results. We believe, therefore, that our estimated concordance rate of $36 \%$ is likely to be an overestimate of the true concordance rate.

Twin pairs who are under the age of 15 at the time of diagnosis, and those who possess HLA-DR 3 and 4, seem to have an increased rate of concordance for Type 1 diabetes [13]. Although our group of twins seems to be representative of Type 1 diabetic patients with respect to their age and HLA type, numbers are not large enough to determine concordance for these two variables independently.

Differences between identical twins must be due to causes other than the germ-line genes. However, even identical twins could be genetically different for certain genes; for example, T-cell receptor and immunoglobulin molecules result from both random and ordered recombination of the genes encoding them and may differ between identical twins [14]. The low concordance rate the we have found suggests that a nongenetically determined event due either to an environmental agent or a random event leading to somatic recombination of certain genes is important in the pathogenesis of Type 1 diabetes.

The non-genetically determined factor causing Type 1 diabetes could operate over a finite, or a prolonged, period. The disease rate in the co-twins fell rapidly in the years after the diagnosis of the index twin, which suggests that the initiating process leading to B-cell destruction occurs over a finite, rather than a prolonged, period. The pattern of incidence of Type 1 diabetes in the general population rises to a peak before 15 years of age and falls sharply thereafter, consistent with exposure or susceptibility being limited to a relatively brief period [15]. Lack of clustering of diabetes in populations suggests that the critical period is more likely to represent susceptibility to an environmental agent than exposure to that agent [16]. The critical process causing Type 1 diabetes is unlikely to start at any specific age as the incidence of the disease is not log-normally distributed (a characteristic of 
point-source exposures). Neither is it likely to occur at random ages since the incidence of the disease is not uniform throughout life. As the disease incidence peaks during childhood, the critical period must usually be early in life.

Acknowledgements. This study has been supported by the Medical Research Council and the Nuffield Foundation. RDGL and DAH are supported by the Wellcome Trust. We are indebted to the following research fellows who have studied our diabetic identical twin pairs: Drs. J.Cassar, R. B. Tattersall, P.G. Nelson, C. Theophanides, A.H.Barnett, C.Johnston and P.J.Hoskins. We also thank Mrs. A. Spinks for secretarial help.

\section{References}

1. Marks HH, Krall LP, White P (1971) Epidemiology and detection of Diabetes. In: Joslin EP, Root H, White P, Marble A (eds) Treatment of diabetes mellitus, 11th edn. Lea and Febinger, Philadelphia, pp 10-34

2. Gottlieb MS, Root HF (1968) Diabetes mellitus in twins. Diabetes 17: 693-704

3. Harvald B, Hauge M (1963) Selection in diabetes in modern society. Acta Med Scand 173: 459-465

4. Barnett AH, Eff C, Leslie RDG, Pyke DA (1981) Diabetes in identical twins: a study of 200 pairs. Diabetologia 20:87-93

5. Millward BA, Welsh KI, Leslie RDG, Pyke DA, Demaine AG (1987) $\mathrm{T}$ cell receptor beta chain polymorphisms are associated with insulin-dependent diabetes. Clin Exp Immunol 70: 152-157

6. Platz P, Jacobsen BK, Morling N, Ryder LP, Svejgaard A, Thomson $\mathbf{M}$, Christy $M$, Kromann $M$, Benn $J$, Nerup $J$, Green A, Hauge $M$ (1981) HLA-D and -DR antigens in genetic analysis of insulin-dependent diabetes mellitus. Diabetologia 21: 108-115

7. Pak CY, Hyone-Myong E, McArthur RG, Yoon JW (1988) Association of cytomegalovirus infection with autoimmune Type 1 diabetes. Lancet II: 1-4
8. Peto R (1977) In: Hiatt HH, Watson JD, Winstein JA (eds) Origins of human cancer. Cold Spring Harbor, New York

9. National Diabetes Data Group (1979) Classification and diagnosis of diabetes mellitus and other categories of glucose intolerance. Diabetes 28: 1039-1057

10. Elandt-Johnson RL, Johnson NL (1980) Survival models and data analysis. John Wiley and Sons, New York

11. Bidwell JL, Bidwell EA, Laundy GJ, Klouda PT, Bradley BA (1987) Allegenotypes defined by short DQ alpha and DQ beta cDNA probes correlate with and define splits of HLA-DQ serological specificities. Mol Immunol 24: 513-522

12. Tarn AC, Thomas JM, Dean BM, Ingram D, Schwartz G, Bottazzo GF, Gale EAM (1988) Predicting insulin-dependent diabetes. Lancet I: 845-850

13. Johnston C, Pyke DA, Cudworth AG, Wolf E (1983) HLA-DR typing in identical twins with insulin-dependent diabetes: a difference between concordant and discordant pairs. Br Med J 253-255

14. Tonegawa S (1983) Somatic generation of antibody diversity. Nature $302: 575$

15. Christau B, Kromann H, Christy M, Ortved Andersen O, Nerup $\mathrm{J}$ (1979) Incidence of insulin-dependent diabetes mellitus (0-29 years at onset) in Denmark. Acta Med Scand [Suppl] 624: $54-60$

16. Gamble DR (1980) The epidemiology of insulin dependent diabetes, with particular reference to the relationship of virus infection to its etiology. Epidemiol Rev 2: 49-70

Received: 6 June 1988

and in revised form: 15 August 1988

Dr. R. D.G. Leslie

Kings College Hospital

Denmark Hill

London SE5 8RX

UK 